Pacific Journal of Mathematics

HOMOMORPHISMS OF COMMUTATIVE RINGS WITH UNIT 


\title{
HOMOMORPHISMS OF COMMUTATIVE RINGS WITH UNIT ELEMENT
}

\author{
E. FRIED AND J. SiChLER
}

Let $R$ be a commutative ring. All its endomorphisms form a monoid $\mathscr{E}(R)$ and a natural question to ask is what monoids appear as full endomorphism monoids of commutative rings. It was shown in [8] that every group is representable as the full automorphism group of a ring without unit element. Much more cannot be expected in this case as the zero mapping is always one of the endomorphisms. The presence of the unit element 1 in the ring changes the picture. We will show here that every monoid is isomorphic to the monoid $\mathscr{E}_{1}(R)$ of all 1-preserving endomorphisms of a commutative ring $R$ with 1 . In fact, a stronger theorem will be proved: the category $\mathscr{R}_{1}$ of all rings with 1 and all 1 preserving homomorphisms is binding.

Definition. A category $\mathscr{C}$ is binding if every category of algebras is isomorphic to a full subcategory of $\mathscr{C}$.

Every monoid is representable as $\operatorname{Hom}_{\mathscr{C}}(C, C)$ for a suitable object $C$ of a binding category $\mathscr{C}$; see e.g. [3]. Many other properties are also shared by binding categories. There is a considerable list of binding categories: categories of directed [5] and undirected graphs [7], the category of semigroups [3], the category of commutative groupoids [9], the category of bounded lattices [1], and other categories of algebras. Next is the list of theorems proved here.

\section{Full Embedding Theorem. $\mathscr{R}_{1}$ is binding.}

This is the basic theorem. The remaining theorems are consenquences of results proved elsewhere and of the proof of the above theorem.

RePResentation TheOREM. Let $M$ be a monoid, let $\sigma$ be a cardinal number, $\sigma \geqq \max \left(\boldsymbol{\aleph}_{0},|\boldsymbol{M}|\right)$. Then there is a set $\left(R_{\alpha} \mid \alpha \in 2^{\sigma}\right)$ of commutative rings $R_{\alpha}$ with unit such that for all $\alpha, \alpha^{\prime} \in 2^{\sigma}$.

(i) $\left|R_{\alpha}\right|=\sigma$,

(ii) $\mathscr{E}_{1}\left(R_{\alpha}\right) \cong M$,

(iii) $\operatorname{Hom}_{\mathscr{\Omega}_{1}}\left(R_{\alpha}, R_{\alpha^{\prime}}\right)=\varnothing$ whenever $\alpha \neq \alpha^{\prime}$.

In particular, the rings $R_{\alpha}$ are pairwise nonisomorphic. Note also that the result is the best possible-there are exactly $2^{\circ}$ pairwise nonisomorphic rings of a cardinality $\sigma \geqq \aleph_{0}$. 
Subring INDEPENDENCE TheOREM. Let $\boldsymbol{M}_{1}$ and $\boldsymbol{M}_{2}$ be monoids. Then there are commutative rings with unit $R_{1}$ and $R_{2}$ such that $R_{1}$ is a subring of $R_{2}$ and $\mathscr{E}_{1}\left(R_{i}\right) \cong M_{i}$ for $i=1,2$.

Quotient RIng Independence Theorem. Let $\boldsymbol{M}_{1}$ and $\boldsymbol{M}_{2}$ be monoids. Then there are commutative rings with unit $R_{1}$ and $R_{2}$ such that $R_{2}$ is a homomorphic image of $R_{1}$ and $\mathscr{E}_{1}\left(R_{i}\right) \cong \boldsymbol{M}_{i}$ for $i=$ $1,2$.

Extension Property. Let $M$ be a monoid of transformations on the set $X$. Then there is a commutative ring with unit $R$ such that $R$ contains $X$ and every $m \in M$ extends uniquely to an endomorphism of $R$. This extension is an isomorphism between $M$ and $\mathscr{E}_{1}(R)$.

To prove the first theorem a full embedding $\Phi$ of the category $\mathscr{G}$ of undirected graphs into $\mathscr{R}_{1}$ will be constructed in third section. The necessary definitions follow.

2. Graphs and categories. An undirected graph $G$ is a pair $G=\langle X, R\rangle$ where $X$ is a set and $R$ is a set of two-element subsets of $X$. Let $G^{\prime}=\left\langle X^{\prime}, R^{\prime}\right\rangle$ be another graph; a mapping $f: X \rightarrow X^{\prime}$ is compatible if $\left\{x_{1}, x_{2}\right\} \in R$ implies $\left\{f\left(x_{1}\right), f\left(x_{2}\right)\right\} \in R^{\prime}$. Let $\mathscr{G}$ be the category whose objects are all undirected graphs and whose morphisms are all compatible mappings. A morphism $f: G \rightarrow G^{\prime}$ is onto if $f$ itself is an onto mapping and if $R^{\prime}=\left\{\left\{f\left(x_{1}\right), f\left(x_{2}\right)\right\} \mid\left\{x_{1}, x_{2}\right\} \in R\right\}$.

A concrete category is a category $\mathscr{C}$ together with a fixed faithful functor $U: \mathscr{C} \rightarrow$ Set (Set is the category all sets and all mappings). $\mathscr{G}$ is concrete category with $U(\langle X, R\rangle)=X$; for categories of algebras we shall always choose the standard underlying-set functor. Let $\left\langle\mathscr{C}_{1}, U_{1}\right\rangle$ and $\left\langle\mathscr{C}_{2}, U_{2}\right\rangle$ be two concrete categories. A functor $\Sigma: \mathscr{C}_{1} \rightarrow$ $\mathscr{C}_{2}$ is a full embedding if $\Sigma$ is one-to-one both on objects and on morphisms and if for every $\beta: \Sigma(C) \rightarrow \Sigma\left(C^{\prime}\right)$ there exists a morphism $\alpha: C \rightarrow C^{\prime}$ in $\mathscr{C}_{1}$ such that $\Sigma(\alpha)=\beta$. A full embedding $\Sigma$ is called an extension if there is a monotransformation $\mu: U_{1} \rightarrow U_{2} \circ \Sigma$.

The starting point is the following theorem; it can be easily obtained using results of [5] and [7].

THEOREM A. Let $\mathscr{A}$ be a full category of algebras or a full category of relational systems. Then there is an extension $\Psi_{\mathscr{A}}: \mathscr{C} \rightarrow \mathscr{G}$

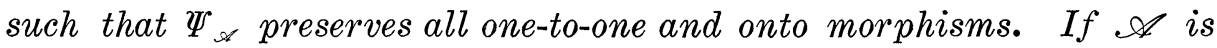
the category of commutative groupoids, then $\Psi_{\infty}$ also preserves the cardinalities of those underlying sets that are infinite.

3. The full embedding. An extension 
will be constructed here.

Let $G=\langle X, R\rangle$ be an undirected graph, let $Z$ be the ring of integers. Consider the ideal $I$ generated by the set $\left\{x^{3}-5.7 \mid x \in X\right\}$ in the polynomial ring $Z[X]$ and let

$$
R(X)=Z[X] / I \text {. }
$$

Obviously, $Z \subseteq R(X)$ and $R(X)$ contains a copy of the set $X$ as a set of generators. Let $\Phi(G)$ be the subring of $R(X)$ generated by the set

$$
Z \cup\{5 x \mid x \in X\} \cup\{x y \mid\{x, y\} \in R\} .
$$

Every compatible mapping $f: G \rightarrow G^{\prime}=\left\langle X^{\prime}, R^{\prime}\right\rangle$ extends uniquely to a 1-preserving homomorphism $\bar{f}: Z[X] \rightarrow Z\left[X^{\prime}\right]$ such that $\bar{f}(I) \subseteq I^{\prime}$. Hence there is a unique homomorphism $R(f): R(X) \rightarrow R\left(X^{\prime}\right)$ such that $R(f)(5 x)=5 f(x)$ and for each $x y$ in $\Phi(G) R(f)(x y)=f(x) f(y)$. As $f$ is a compatible mapping, $R(f)(\Phi(G)) \subseteq \Phi\left(G^{\prime}\right)$. Let $\Phi(f)$ be the restriction of $R(f)$ to $\Phi(G)$; it is easy to see that $\Phi$ is naturally equivalent to a one-to-one functor denoted also as $\Phi$.

The mappings $\mu_{G}: X \rightarrow \Phi(G)$ defined by $\mu_{G}(x)=5 x$ form a natural transformation $\mu: U_{1} \rightarrow U_{2} \circ \Phi$, where $U_{1}: \mathscr{G} \rightarrow$ Set and $U_{2}: \mathscr{R}_{1} \rightarrow$ Set are the standard underlying-set functors. $\mu$ is a monotransformation. To prove that $\Phi$ is an extension it is enough to show that for every 1-preserving homomorphism $g: \Phi(G) \rightarrow \Phi\left(G^{\prime}\right)$ there is a compatible mapping $f: G \rightarrow G^{\prime}$ such that $g=\Phi(f)$.

First of all, adjoin a third root $\rho$ of the unit to $R(X)$; that is, let $\rho^{2}+\rho+1=0$ and let

$$
E(X)=(R(X))[\rho] .
$$

Let $\boldsymbol{E}=\boldsymbol{Z}[\rho]$. Observe that $\boldsymbol{E}(X)$ is a free $\boldsymbol{E}$-module over the set of all commutative products

$$
\pi=x_{1}^{i_{1}} \ldots x_{n}^{i_{n}}
$$

of powers of pairwise different elements of $X$ with $1 \leqq i_{j} \leqq 2$.

In particular, if

$$
e=\sum_{i=1}^{m} e_{i} \pi_{i}
$$

is an element of $E(X)$ such that the products $\pi_{i}$ are pairwise different and if $e$ is divisible by an integer $k$, then $k$ divides every $e_{i}$. No integer is a zero divisor in $\boldsymbol{E}(X)$.

For a product of the form (4) denote

$$
u(\pi)=\left\{x_{1}, \cdots, x_{n}\right\}
$$


and

$$
l(\pi)=|u(\pi)|
$$

Note that always $u\left(\pi_{1} \cdot \pi_{2}\right) \subseteq u\left(\pi_{1}\right) \cup u\left(\pi_{2}\right)$; if $u\left(\pi_{1} \cdot \pi_{2}\right) \neq u\left(\pi_{1}\right) \cup$ $u\left(\pi_{2}\right)$, then the product $\pi_{1} \cdot \pi_{2}$ is divisible by 5.7 .

LEMMA 1. $\eta^{3}=5^{4} \cdot 7$ in $\boldsymbol{E}(X)$ if and only if $\eta=5 \rho^{\alpha} x$ for some $\alpha \in\{0,1,2\}$ and $x \in X$.

Proof. For each $x$ and $\alpha\left(5 \rho^{\alpha} x\right)^{3}=5^{3} \cdot 1 \cdot x^{3}=5^{4} \cdot 7$. Conversely, let $\eta \in \boldsymbol{E}(X)$ and $\eta^{3}=5^{4} \cdot 7 . \quad \eta=\eta\left(x_{1}, \cdots, x_{n}\right)$ for a finite set $\left\{x_{1}, \cdots, x_{n}\right\} \subseteq$ $X$ so it is enough to prove the lemma for finitely generated rings $E_{n}=\boldsymbol{E}\left(\left\{x_{1}, \cdots, x_{n}\right\}\right)$. We will proceed by induction.

Since $E_{1}$ is an integral domain, the polynomial

$$
\eta^{3}-5^{4} \cdot 7
$$

has at most three roots in $E_{1}$. $E_{1}$ contains, however, three different roots of (8), namely $5 x_{1}, 5 \rho x_{1}$ and $5 \rho^{2} x_{1}$.

Assume the lemma to be true for $n$ and let $\eta \in E_{n+1}$ be a root of (8),

$$
\eta=a+b x_{n+1}+c x_{n+1}^{2}
$$

for some $a, b, c$ in $E_{n}$. It is easy to see that mappings

$$
\varphi_{i}: E_{n+1} \rightarrow E_{n+1} \quad(i=0,1,2)
$$

defined by

$$
\varphi_{i}\left(p\left(x_{1}, \cdots, x_{n}, x_{n+1}\right)\right)=p\left(x_{1}, \cdots, x_{n}, \rho^{i} x_{1}\right)
$$

(the coefficients of $p$ are in $\boldsymbol{E}$ ) are endomorphisms of $E_{n+1}$ and that $\varphi_{i}\left(E_{n+1}\right)=E_{n}$ for $i=0,1,2$. Put

$$
\eta_{i}=\varphi_{i}(\eta) \quad(i=0,1,2) .
$$

Clearly

$$
\begin{gathered}
\eta_{0}=a+b x_{1}+c x_{1}^{2}, \\
\eta_{1}=a+b \rho x_{1}+c \rho^{2} x_{1}^{2}, \\
\eta_{2}=a+b \rho^{2} x_{1}+c \rho x_{1}^{2},
\end{gathered}
$$

and $\eta_{0}, \eta_{1}, \eta_{2}$ are roots of (8) in $E_{n}$. By the induction hypothesis $\eta_{0}=$ $5 \rho^{\alpha} x_{i}, \eta_{1}=5 \rho^{\beta} x_{j}, \eta_{2}=5 \rho^{\gamma} x_{k}$ for some $\alpha, \beta, \gamma \in\{0,1,2\}$ and $i, j, k \in$ $\{1, \cdots, n\}$. Consequently, $3 c x_{1}^{2}=\eta_{0}+\rho \eta_{1}+\rho^{2} \eta_{2}=5 \rho^{\alpha} x_{i}+5 \rho^{\beta+1} x_{j}+5 \rho^{\gamma+2} x_{k}$. Since the right side of the last equation is divisible by three, $x_{i}=x_{j}=$ 
$x_{k}$; so $3 c x_{i}^{2}=5\left(\rho^{\alpha}+\rho^{\beta+1}+\rho^{\gamma+2}\right) x_{i}$. Multiplying both sides by $x_{1}$ and dividing by five yields $7(3 c)=\left(\rho^{\alpha}+\rho^{\beta+1}+\rho^{\gamma+2}\right) x_{1} x_{i}$. A sum of three third roots of the unit is divisible by seven if and only if it vanishes, i.e., if either $\alpha \equiv \beta \equiv \gamma(\bmod 3)$ or $\beta \equiv \alpha+1(\bmod 3)$ and $r \equiv \alpha+2(\bmod 3)$. In both cases $c=0$.

Let $\alpha \equiv \beta \equiv \gamma(\bmod 3)$. Note that $3 \alpha=\eta_{0}+\eta_{1}+\eta_{2}=5\left(\rho^{\alpha}+\rho^{\beta}+\rho^{\gamma}\right) x_{i}$; therefore $a=5 \rho^{\alpha} x_{i}$ and $b=0$.

If $\beta \equiv \alpha+1(\bmod 3)$ and $\gamma \equiv \alpha+2(\bmod )$ then $a=0$. As $c=0$, (11) implies $b x_{1}=5 \rho^{\alpha} x_{i}$. If $x_{1} \neq x_{i}$, then $7 b=\rho^{\sigma} x_{i} x_{1}^{2}$-a contradiction. Therefore $x_{i}=x_{1}$ and $b=5 \rho^{\alpha}$.

Since $\boldsymbol{E}(X)=R(X)[\rho]$ the only roots of (8) in $R(X)$ are of the form

$$
\eta=5 x \quad(x \in X) .
$$

All of them are contained in $\Phi(G)$.

Lemia 2. The only idempotent elements in $\Phi(G)$ are 0 and 1. $s^{3}=0$ in $\Phi(G)$ if and only if $s=0$.

Proof. As $\Phi(G)$ is a subring of $\boldsymbol{E}(X)$, we need only prove the lemma for $\boldsymbol{E}(X)$; in fact the proof for all the rings $E_{n}$ is sufficient. We can proceed by induction again. $E_{1}$ is an integral domain, therefore both assertions hold there. The rest of the proof is similar to the proof of Lemma 1.

Lemma 3. Let $G=\langle X, R\rangle$ be a graph. A product $x y(x, y \in X)$ belongs to $\Phi(G)$ if and only if $\{x, y\} \in R$.

Proof. If $\{x, y\} \in R$, then $x y \in \Phi(G)$ by definition.

Conversely let $S$ be the set of all elements $\sigma$ of $R(X)$ of the form

$$
\sigma=k+\sum_{u(\pi) \in R} k_{\pi} \cdot \pi+\sum m_{\varphi} \cdot \varphi
$$

where $k, k_{\tilde{\tau}} \in\{0, \cdots, 4\}$ and for every $\varphi$ either $l(\varphi)>2$ or $m_{\varphi}$ is divisible by five. All the generators of $\Phi(G)$ are of the form (15) and it is easy to see that $S$ is a ring; $R(X) \supseteqq S \supseteqq \Phi(G)$. Since $R(X)$ is a free abelian group over the set of all products of the form (4), (15) is determined by $\sigma$ uniquely. The lemma follows.

To finish the proof of fullness of $\Phi$, let $g: \Phi(G) \rightarrow \Phi\left(G^{\prime}\right)$ be a 1preserving homomorphism and let $x \in X . \quad(g(5 x))^{3}=g\left((5 x)^{3}\right)=g\left(5^{4} \cdot 7\right)=$ $5^{4} \cdot 7$, thus, by Lemma $1, g(5 x)=5 f(x)$ for some $f(x) \in X^{\prime}\left(f: X \rightarrow X^{\prime}\right.$ is a well-defined mapping). Let $\{x, y\} \in R$. By the definition of $\Phi(G)$, 
$x y \in \Phi(G)$ and $25 g(x y)=g(5 x \cdot 5 y)=g(5 x) \cdot g(5 y)=25 f(x) f(y)$, so $g(x y)=$ $f(x) f(y)$. The product $f(x) \cdot f(y)$ belongs to $\Phi\left(G^{\prime}\right)$. By Lemma 3, $\{f(x), f(y)\} \in R^{\prime} . \quad f:\langle X, R\rangle \rightarrow\left\langle X^{\prime}, R^{\prime}\right\rangle$ is a morphism in $\mathscr{G}$ and $\Phi(f)=$ $g$-proving the fullness of $\Phi$.

Now, let $h: \Phi(G) \rightarrow \Phi\left(G^{\prime}\right)$ be a homomorphism not preserving the unit element 1 . Since 0 is the only other idempotent in $\Phi\left(G^{\prime}\right), h(1)=0$. Thus $h(n)=0$ for every integer $n$ and, in particular, $(h(5 x))^{3}=$ $h\left((5 x)^{3}\right)=h\left(5^{4} \cdot 7\right)=0$ and $(h(x y))^{3}=h\left(x^{3} y^{3}\right)=h\left(5^{2} \cdot 7^{2}\right)=0$. According to Lemma 2, $h(5 x)=0$ a $h(x y)=0$. All generators of $\Phi(G)$ are mapped to the zero of $\Phi\left(G^{\prime}\right)$ so $h$ is the zero homomorphism. The last observation is utilized as follows.

THEOREM. Let $\mathscr{S}$ be the category of all commutative rings with unit 1 and all their (not necessarily 1-preserving) homomorphisms. Let $\mathscr{N}$ be the class of all nonzero homomorphisms of $\mathscr{S}$. Then there is a full subcategory $\mathscr{F}$ of $\mathscr{S}$ such that

(i) $\mathscr{F} \cap \mathscr{N}$ is a category

(ii) $\mathscr{F} \cap \mathscr{N}$ is binding.

In particular, for every monoid $M$ there is a commutative ring $R$ with unit such that the set of all its nonzero endomorphisms is closed under composition and isomorphic to $\boldsymbol{M}$. All the theorems listed in the first section can be similarly reformulated.

4. Concluding remarks. First we shall indicate the proofs of the remaining four theorems.

The Representation Theorem is an immediate consequence of the proof of the Full Embedding Theorem, Theorem A and Theorem 4 of [6].

To prove the Subring Independence and Quotient Ring Independence Theorems, observe that the extension $\Phi: \mathscr{P} \rightarrow \mathscr{R}_{1}$ preserves all one-to-one and all onto morphisms. Combining this fact with Theorem $\mathrm{A}$ and the main results [4] and [2] respectively, we obtain both Independence Theorems.

The proof of the Extension Property is based on the fact that there is an extension $\Phi \circ \Psi: \mathscr{A} \rightarrow \mathscr{R}_{1}$ for every category $\mathscr{A}$ of relational systems (Theorem A and the third section) and on the observation that every monoid $M$ of transformations on the set $X$ is the monoid of all mappings $X \rightarrow X$ compatible with one $|X|$-ary relation.

L. Kučera and Z. Hedrlín have proved recently that any concrete category has an extension to $\mathscr{C}$ provided there are no measurable cardinals. Using this fact one can generalize immediately the Exten- 
sion Property to the statement saying that any concrete category has an extension to the category of all commutative rings with 1 and all 1-preserving homomorphisms (under the hypothesis of nonexistence of measurable cardinals).

We conclude by mentioning two open problems. Note that all rings $\Phi(G)$ have zero divisors and are infinite. Thus the present results do not apply to the case of finite rings and to the case of integral domains.

\section{REFERENCES}

1. G. Grätzer and J. Sichler, On the endomorphism semigroup (and category) of bounded lattices. Pacific J. Math., 35 (1970), 639-647.

2. Z. Hedrlín, On endomorphisms of graphs and their homomorphic images, Proof Techniques in Graph Theory, Ed. F. Harrary, Academic Press, 1969.

3. Z. Hedrlín and J. Lambek, How comprehensive is the category of semigroups? J. Algebra, 11 (1969), 195-212.

4. Z. Hedrlín and E. Mendelsohn, The category of graphs with given subgraph, Canad. J. Math., 21 (1969), 1506-1517.

5. Z. Hedrlín and A. Pultr, On full embeddings of categories of algebras, Illinois J. Math., 10 (1966), 392-405.

6. Z. Hedrlín and J. Sichler, Any boundable binding category contains a proper class of mutually disjoint copies of itself, Algebra Universalis 1, (1971), 97-103.

7. P. Hell, Full embeddings into some categories of graphs, to appear.

8. R. Quackenbush and J. Sichler, Automorphism groups of commutative rings, to appear.

9. J. Sichler, Category of commutative groupoids is binding, Comment. Math. Univ. Carolinae, 8 (1967), 753-755.

Received June 15, 1971. The authors were supported by grants from National Research Council of Canada.

The University of Manitoba 



\section{PACIFIC JOURNAL OF MATHEMATICS}

\section{EDITORS}

\author{
H. SAMELson \\ Stanford University \\ Stanford, California 94305 \\ C. R. HоввY \\ University of Washington \\ Seattle, Washington 98105
}

J. DugundJI

Department of Mathematics

University of Southern California

Los Angeles, California 90007

RICHARD ARENS

University of California

Los Angeles, California 90024

\section{ASSOCIATE EDITORS}
E. F. BeCKENBACH
B. H. NEUMANN
F. WOLF
K. YoshidA

\section{SUPPORTING INSTITUTIONS}

UNIVERSITY OF BRITISH COLUMBIA

CALIFORNIA INSTITUTE OF TECHNOLOGY

UNIVERSITY OF CALIFORNIA

MONTANA STATE UNIVERSITY

UNIVERSITY OF NEVADA

NEW MEXICO STATE UNIVERSITY

OREGON STATE UNIVERSITY

UNIVERSITY OF OREGON

OSAKA UNIVERSITY

\author{
UNIVERSITY OF SOUTHERN CALIFORNIA \\ STANFORD UNIVERSITY \\ UNIVERSITY OF TOKYO \\ UNIVERSITY OF UTAH \\ WASHINGTON STATE UNIVERSITY \\ UNIVERSITY OF WASHINGTON

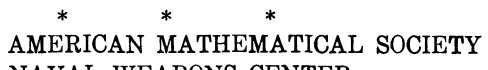 \\ NAVAL WEAPONS CENTER
}

The Supporting Institutions listed above contribute to the cost of publication of this Journal, but they are not owners or publishers and have no responsibility for its content or policies.

Mathematical papers intended for publication in the Pacific Journal of Mathematics should be in typed form or offset-reproduced, (not dittoed), double spaced with large margins. Underline Greek letters in red, German in green, and script in blue. The first paragraph or two must be capable of being used separately as a synopsis of the entire paper. The editorial "we" must not be used in the synopsis, and items of the bibliography should not be cited there unless absolutely necessary, in which case they must be identified by author and Journal, rather than by item number. Manuscripts, in duplicate if possible, may be sent to any one of the four editors. Please classify according to the scheme of Math. Rev. Index to Vol. 39. All other communications to the editors should be addressed to the managing editor, Richard Arens, University of California, Los Angeles, California, 90024.

50 reprints are provided free for each article; additional copies may be obtained at cost in multiples of 50 .

The Pacific Journal of Mathematics is issued monthly as of January 1966. Regular subscription rate: $\$ 48.00$ a year (6 Vols., 12 issues). Special rate: $\$ 24.00$ a year to individual members of supporting institutions.

Subscriptions, orders for back numbers, and changes of address should be sent to Pacific Journal of Mathematics, 103 Highland Boulevard, Berkeley, California, 94708.

PUBLISHED BY PACIFIC JOURNAL OF MATHEMATICS, A NON-PROFIT CORPORATION

Printed at Kokusai Bunken Insatsusha (International Academic Printing Co., Ltd.), 270, 3-chome Totsuka-cho, Shinjuku-ku, Tokyo 160, Japan. 


\section{Pacific Journal of Mathematics}

\section{Vol. 45, No. $2 \quad$ October, 1973}

Kenneth Paul Baclawski and Kenneth Kapp, Induced topologies for quasigroups and loops ............................................. 393

D. G. Bourgin, Fixed point and $\min -\max$ theorems $\ldots \ldots \ldots \ldots \ldots \ldots \ldots$

J. L. Brenner, Zolotarev's theorem on the Legendre symbol ............... 413

Jospeh Atkins Childress, Jr., Restricting isotopies of spheres .............. 415

John Edward Coury, Some results on lacunary Walsh series ................ 419

James B. Derr and N. P. Mukherjee, Generalized Sylow tower groups. II . . . . . . 427

Paul Frazier Duvall, Jr., Peter Fletcher and Robert Allen McCoy, Isotopy Galois

spaces .......................................... 435

Mary Rodriguez Embry, Strictly cyclic operator algebras on a Banach space ... 443

Abi (Abiadbollah) Fattahi, On generalizations of Sylow tower groups ......... 453

Burton I. Fein and Murray M. Schacher, Maximal subfields of tensor products . . 479

Ervin Fried and J. Sichler, Homomorphisms of commutative rings with unit

element .......................................... 485

Kenneth R. Goodearl, Essential products of nonsingular rings ............. 493

George Grätzer, Bjarni Jónsson and H. Lakser, The amalgamation property in

equational classes of modular lattices ...........................

507

$\mathrm{H}$. Groemer, On some mean values associated with a randomly selected simplex

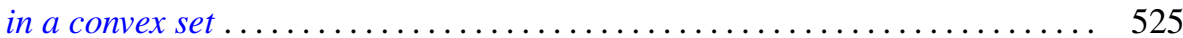

Marcel Herzog, Central 2-Sylow intersections .................... 535

Joel Saul Hillel, On the number of type-k translation-invariant groups ........ 539

Ronald Brian Kirk, A note on the Mackey topology for $\left(C^{b}(X)^{*}, C^{b}(X)\right) \ldots \ldots .543$

J. W. Lea, The peripherality of irreducible elements of lattice.............. 555

John Stewart Locker, Self-adjointness for multi-point differential operators ..... 561

Robert Patrick Martineau, Splitting of group representations ............... 571

Robert Massagli, On a new radical in a topological ring ................. 577

James Murdoch McPherson, Wild arcs in three-space. I. Families of Fox-Artin

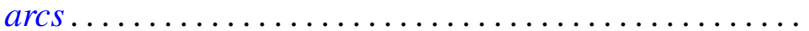

585

James Murdoch McPherson, Wild arcs in three-space. III. An invariant of

oriented local type for exceptional arcs . . . . . . . . . . . . ............ 599

Fred Richman, The constructive theory of countable abelian p-groups ........ 621

Edward Barry Saff and J. L. Walsh, On the convergence of rational functions

which interpolate in the roots of unity ..................

Harold Eugene Schlais, Non-aposyndesis and non-hereditary

decomposability..................................... 643

Mark Lawrence Teply, A class of divisible modules................... 653

Edward Joseph Tully, Jr., H-commutative semigroups in which each

homomorphism is uniquely determined by its kernel ................. 669

Garth William Warner, Jr., Zeta functions on the real general linear group ...... 681

Keith Yale, Cocyles with range $\{ \pm 1\} \ldots \ldots \ldots \ldots \ldots \ldots \ldots \ldots \ldots \ldots \ldots \ldots \ldots . \ldots \ldots$

Chi-Lin Yen, On the rest points of a nonlinear nonexpansive semigroup ........ 699 